between the two studies. Thus, whereas "current asthma" was apparently categorised according to current medication in the LUNOKID study, within the SLIC study "current asthma" was defined as those with "either doctor diagnosis or asthma medication in the past 12 months, with or without current symptoms/ wheeze", ensuring that any child with a prior history of asthma was only included if asymptomatic and without treatment for at least 12 months. Despite these differences in asthma classification, it is reassuring to know that "no relevant mean differences were found for the other subgroups or for the total study population when all subgroups were included" within the LUNOKID study, thereby confirming our findings from the SLIC study. Both studies also agree on the higher failure rate due to technically unsatisfactory data when including children with respiratory symptoms. However, while the LUNOKID authors argue that due to potential difficulties in separating upper and lower respiratory tract infections, "strict criteria to define a healthy population should be adhered to", our experience suggests that any naïve child (i.e. one unfamiliar with spirometric assessments) with significant respiratory symptoms is likely to be self-excluded provided strict quality control is applied, and that exclusion of a high proportion of the population on "health grounds" could result in the over estimation of abnormalities and potential mismanagement of lung disease.

In conclusion, while we completely agree that inclusion and exclusion criteria applied to subjects must vary according to the underlying question and study design, we would like to confirm that we excluded results from any children with current asthma or who were on asthma medication. For the purposes of data collection in population-based studies of lung function, such as the SLIC study, we stand by our conclusion that with exception of clear cut factors, such as current and chronic respiratory disease, paediatric reference samples for spirometry can be relatively inclusive and hence more generalisable to the general population.

@ERSpublications

Excluding current/chronic lung disease, population samples for children's lung function can be relatively inclusive http://ow.ly/X0enM

Sooky Lum and Janet Stocks

Respiratory, Critical Care and Anaesthesia section (Portex Unit), UCL, Institute of Child Health, London, UK.

Correspondence: Sooky Lum, Respiratory, Critical Care and Anaesthesia section (Portex Unit), UCL, Institute of Child Health, 30 Guilford Street, London, WC1N 1EH, UK. E-mail: s.lum@ucl.ac.uk

Received: Dec 032015 | Accepted after revision: Dec 162015

Conflict of interest: None declared.

\title{
References
}

1 Lum S, Bountziouka V, Sonnappa S, et al. How "healthy" should children be when selecting reference samples for spirometry? Eur Respir J 2015; 45: 1576-1581.

2 Hüls A, Krämer U, Gappa M, et al. Neue Spirometrische Referenzwerte für Kinder und Jugendliche in Deutschland unter berücksichtigung der Größe und nichtlinearer Alterseffekte: tie LUNOKID-Studie [New spirometric reference values for children and adolescents in Germany considering height and non-linear age effects: the LUNOKID-Study]. Pneumologie 2014; 68: 393.

3 Lum S, Bountziouka V, Sonnappa S, et al. Lung function in children in relation to ethnicity, physique and socioeconomic factors. Eur Respir J 2015; 46: 1662-1671.

\section{Interstitial pneumonia with autoimmune features: the new consensus-based definition for this cohort of patients should be broadened}

To the Editor:

We appreciate the research statement recently published by FisCHER et al. [1] proposing new terminology: "interstitial pneumonia with autoimmune features" (IPAF) to characterise the heterogeneous group of 
patients with idiopathic interstitial pneumonia (IIP) who have a clinical flavour of underlying connective tissue disease (CTD) but do not meet the current American College of Rheumatology criteria for CTD. Indeed, a variety of terms and criteria have been used in studies to describe such patients, including "autoimmune featured interstitial lung disease", "undifferentiated connective tissue disease associated interstitial lung disease", and "lung-dominant connective tissue disease" [2-5]. Thus, the need for a consensus regarding the terminology and better characterisation of the clinical characteristics of this group of patients is evident. The proposed terminology, however, is not yet validated and does not include a provision for patients with clinical characteristics of CTD who do not meet American College of Rheumatology criteria for CTD or IPAF criteria, but are distinct from patients with IIP alone. To meet the proposed criteria for IPAF, a patient must have features of IIP on high-resolution computed tomography (HRCT) images and/or surgical lung biopsy. Alternative aetiologies of IIPs must be excluded by thorough clinical evaluation and the patient must fail to meet criteria for a specific CTD. The patient must then have at least one characteristic from at least two out of three domains: clinical, serological and morphological. We wish to share our concerns regarding the new IPAF classification.

Our primary concern with the proposed IPAF criteria is that usual interstitial pneumonia (UIP) is excluded from the morphological domain. This is problematic as UIP is a common pattern of IIP among patients with positive serology for CTD who do not meet criteria for specific CTD and do not meet clinical criteria for IPAF and, thus, are currently labelled as IPF. While this is in keeping with the 2011 criteria for the definition of IPF and may be the reason for excluding UIP in the proposed definition for IPAF, recent studies in patients with lung dominant CTD (defined as patients with positive serologies for CTD in the absence of extrathoracic features of a definite CTD) documented that the UIP pattern was the most common IIP pattern of disease, occurring in $57 \%$ of the 44 patients in this cohort $[4,6,7]$. VIj et al. [2] documented that $62 \%$ of patients with "autoimmune featured ILD" characterised by IIP, positive serologies and one or more symptoms suggestive of CTD but not meeting American College of Rheumatology criteria had a UIP pattern on HRCT. Studies of patients with IIP and autoimmune characteristics have produced different estimates of survival when compared to patients with chronic fibrosing lung disease, which may be related to differences in the prevalence of the UIP pattern between these cohorts $[2,8,9]$. UIP has been associated with poor survival among patients with CTD in some studies, but survival may still be better than that among patients with IPF [7, 10, 11]. These studies highlight the importance of the UIP pattern among these patients, and we strongly believe that UIP should be included in the morphological domain of the IPAF criteria.

We also have concerns regarding the IPAF clinical domain, which is limited to seven criteria. Based on the current IPAF definition, a patient with an abnormal CTD serology, UIP and oesophageal dysmotility and/ or myalgias would not fulfil the proposed criteria for patients with IPAF. We feel that such patients differ from those with IIP that is limited to the lungs and may indeed have subclinical autoimmune disease and/ or later manifest into a specific CTD. Perhaps these patients would be better acknowledged as having "autoimmune ILD"; autoimmune, simply based on positive CTD serology. Indeed, we appreciate that the proposed IPAF classification is intended for research purposes and would presumably lead to the design of clinical trials enrolling patients with IPAF as proposed by Fischer et al. [1]. We are concerned that by excluding UIP from the proposed morphological domain and excluding patients with other clinical features suggestive of subclinical autoimmune diseases, we would be prematurely disregarding such patients, labelling them as IIP (different from IPAF and by default including them into the category of IIP) and IPF if they have UIP. Such patients are arguably in greatest need of research efforts for effective treatments, yet may be excluded and left in the open, meeting neither criteria for IPAF nor IPF if the proposed IPAF criteria are widely applied to future studies/clinical trials.

While we applaud the efforts of the authors in proposing criteria for IPAF and acknowledge that this is a step in the right direction, we strongly believe the criteria should be reconsidered to encompass patients with autoimmune ILD who do not meet the proposed criteria for IPAF, particularly those with UIP, as well as those with positive serologies and subtle/less specific symptoms of CTD.

0 @ERSpublications

IPAF excludes patients with ILD associated with autoimmune features; IPAF morphological domain should be broadened http://ow.ly/X6VSe

Bridget Collins and Ganesh Raghu

Center for Interstitial Lung Disease, University of Washington Medical Center, University of Washington, Seattle, WA, USA.

Correspondence: Ganesh Raghu, Center for Interstitial Lung Diseases, University of Washington Medical Center, 1959 NE Pacific, Campus Box 356175, Seattle, WA 98195, USA E-mail: graghu@uw.edu

Received: Dec 102015 | Accepted after revision: Dec 232015

Conflict of interest: None declared. 


\section{References}

1 Fischer A, Antoniou KM, Brown KK, et al. An official European Respiratory/American Thoracic Society research statement: interstitial pneumonia with autoimmune features. Eur Respir J 2015; 46: 976-987.

2 Vij R, Noth I, Strek ME. Autoimmune-featured interstitial lung disease: a distinct entity. Chest 2011; 140: 1292-1299.

3 Fischer A, West SG, Swigris JJ, et al. Connective tissue disease-associated interstitial lung disease: a call for clarification. Chest 2010; 138: 251-256.

4 Omote N, Taniguchi H, Watanabe N, et al. Lung-dominant connective tissue disease: clinical, radiologic, and histologic features. Chest 2015: 148: 1438-1446.

5 Kinder BW, Shariat C, Collard HR, et al. Undifferentiated connective tissue disease-associated interstitial lung disease: changes in lung function. Lung 2010; 188: 143-149.

6 Raghu G, Collard HR, Egan JJ. An official ATS/ERS/JRS/ALAT statement: idiopathic pulmonary fibrosis: evidence-based guidelines for diagnosis and management. Am J Respir Crit Care Med 2011; 183: 788-824.

7 Kim EJ, Collard HR, King Jr TE. Rheumatoid arthritis associated interstitial lung disease: the relevance of histopathologic and radiographic pattern. Chest 2009; 136: 1397-1405.

8 Assayag D, Kim EJ, Elicker BM, et al. Survival in interstitial pneumonia with features of autoimmune disease: a comparison of proposed criteria. Respir Med 2015; 109: 1326-1331.

9 Corte TJ, Copley SJ, Desai SR, et al. Significance of connective tissue disease features in idiopathic interstitial pneumonia. Eur Respir J 2012; 39: 661-668.

10 Kim EJ, Elicker BM, Maldonado F, et al. Usual interstitial pneumonia in rheumatoid arthritis-associated interstitial lung disease. Eur Respir J 2010; 35: 1322-1328.

11 Park JH, Kim DS, Park IN, et al. Prognosis of fibrotic interstitial pneumonia: idiopathic versus collagen vascular disease-related subtypes. Am J Respir Crit Care Med 2007; 175: 705-711.

From the authors:

We appreciate the careful reading and concerns expressed by Drs. Collins and Raghu regarding our recently published European Respiratory Society/American Thoracic Society Research Statement on "interstitial pneumonia with autoimmune features" (IPAF). Critical assessment of this document and concept is essential to improving our understanding of this novel classification.

The primary criticism expressed in the letter by Collins and Raghu is that the usual interstitial pneumonia (UIP) pattern of disease is not included within the morphological domain, thereby excluding patients with UIP pattern from the IPAF definition. This is an incorrect conclusion that stems from confusion over the role of domains in the IPAF definition. In the IPAF statement, we explicitly state that patients with UIP can be classified as having IPAF. To paraphrase, having a radiological or histopathological UIP pattern does not exclude IPAF but, unlike non-specific interstitial pneumonia, organising pneumonia or lymphocytic interstitial pneumonia patterns, there is no morphological "credit" associated with the UIP pattern. Thus, to be considered as having IPAF, a patient with a UIP pattern on high-resolution computed tomography or by surgical lung biopsy would need to have at least one feature from the other two domains (clinical and serological) or another pulmonary morphologic feature (e.g. unexplained multi-compartment involvement or histopathologic evidence of interstitial lymphoid aggregates with germinal centres and/or diffuse lymphoplasmacytic infiltration). We appreciate the opportunity to clarify this point.

A second criticism expressed was that the clinical features within the IPAF classification scheme are too limiting and that features such as oesophageal dysmotility and myalgias should also be included. This is a valid criticism. We acknowledge in our discussion, "... in the absence of data to inform decision-making, we were left to devise what this panel believes to be a reasonable first draft of criteria that can be readily applied by investigators who wish to study this interesting, and presently poorly defined, group of patients."

Finally, Collins and Raghu suggest that the term "autoimmune ILD” is more appropriate for this condition. As was explained in our document, the Task Force believed it was important to use straightforward nomenclature to describe an interstitial pneumonia that has certain clinical, serological and/or pulmonary morphological features suggesting the presence of an autoimmune process. We believe the term IPAF achieves this goal.

We are optimistic that the proposed IPAF definition and classification allows for the future study of a uniform cohort of patients with interstitial pneumonia and features of an underlying autoimmune disease. The comments of Collins and Raghu provide an opportunity for us to re-emphasise that prospective studies are needed to validate and refine the proposed criteria, and to determine the natural history and clinical implications of such a diagnosis. 